\author{
${ }^{1}$ Yu.V. Arkhipov, ${ }^{1}$ A. Askaruly, ${ }^{1}$ A.E. Davletov, \\ ${ }^{1}$ D. Dubovtsev ${ }^{*}{ }^{1}$ L. Yerimbetova and ${ }^{2}$ I.M. Tkachenko \\ ${ }^{1}$ IETP, al-Farabi Kazakh National University, al-Farabi71, \\ 050040 Almaty, Kazakhstan \\ ${ }^{2}$ Instituto de Matematica Pura y Aplicada, Universidad Politecnica de Valencia, \\ Camino de Vera s/n, 46022Valencia, Spain \\ *e-mail: denis.dubovcev@gmail.com

\section{Effective potential and pressure of two-component plasma at high temperature}

\begin{abstract}
An effective pair interaction potential of particles for ideal and weakly non-ideal plasma were obtained. It takes into account not only screening and dynamic quantum effects, but also statistical quantum effects. Comparison between other kinds of potentials was conducted. The results for this potential show that our potential tends to a finite value at small distances and lies below Deutsch and Kelbg potentials. At large distances it tends to zero as DebyeHuckel potential. We used this potential for the calculation of plasma pressure and compared our results with simulation data. The calculated pressure values are in a good agreement with simulation one at high temperatures. Key words: pseudo potential, dielectric response formalism, ideal plasma, shielding.
\end{abstract}

\section{Introduction}

It is known, that the interaction of charged particles in plasmas is different from the Coulomb interaction. If we use Coulomb potential, we will have problems with the divergence of collision integrals at scattering angles, because this potential does not take into account the collective effects (screening and quantum effects, for example). In order to overcome these problems, some kinds of potentials (effective potentials), which take into account the collective effects, were introduced to the plasma physics. Let us consider the main ones. Potential of the self-consistent field (Debye Huckel potential corresponds to the approximation of pair correlations and it is applicable for small plasma densities. Deutsch potential takes into account quantum mechanical effects of diffraction and symmetry. Special effective potential, based on the theory of perturbations, was proposed in the paper [1], it is so called Kelbg potential. Deutsch and Kelbg potentials take into account only quantum effects. Authors of [1] proposed a potential which takes into account both screening and quantum mechanical effects.

\section{Effective potential}

The purpose of this article is to show how an effective potential of charged particles interaction can be used for calculating of plasma pressure. This effective potential can be obtained in the framework of the linear dielectric response theory. The main idea is as follows. We chose some microscopic potential, in which we want to add a new property and determine its Fourier transform. Next, the Fourier-image of new potential is determined via the ratio of Fourier-images of microscopic potential and model of dielectric function. Finally, new effective potential with necessary properties is obtained by reverse Fourier transformation. Let's see this scheme in practice:

The equation of this potential in Fourier space then:

$$
\varphi_{\mathrm{ab}}(\mathrm{q})=\phi_{\mathrm{ac}}(\mathrm{q}) \varepsilon_{\mathrm{cb}}^{-1}(\mathrm{q}, 0)
$$

where $\varepsilon_{c b}^{-1}(q, 0)$ is the static dielectric function (SDF) of the plasma, $\phi_{a c}(q)$ is the Fourier transform of a micropotential and $\varphi_{a b}(q)$ - Fourier transform of the desired potential looked for. 
Further, it is necessary to select a micropotential which possesses a part of the required properties, as well as a model for SDF, which has other required properties.

As one of this micro potentials, Deutsch potential were chosen, which takes into account dynamic quantum-mechanical effects (without symmetric part). Its Fourier transform:

$$
\phi(q)=\left(\frac{4 \pi \alpha}{q^{2}}-\frac{4 \pi \alpha}{k_{a b}^{2}+q^{2}}\right) .
$$

A model for the SDF was taken from [2]:

$$
\varepsilon(q, 0)=1+\frac{1}{\left(q / k_{D}\right)^{2}+\left(q / k_{q}\right)^{4}}
$$

Here $\quad k_{D}^{2}=4 e^{2} \beta n_{\text {tot }}, n_{\text {tot }}=n_{e}+\sum_{i} n_{i} Z_{i}^{2}$, $k_{q}^{4}=16 \pi e^{2} / h^{2}\left(n_{e} m_{e}+\sum_{i} n_{i} m_{i} Z_{i}^{2}\right)$.

Now, using these formulas, it is necessary to obtain the equation for the potential in ordinary space by inverse transform of equation (2) using the formula:

$$
\varphi_{a b}(r)=\frac{1}{(2 \pi)^{3}} \int \boldsymbol{d} \boldsymbol{k} \varphi_{a b}(q) \exp (i \boldsymbol{q} \boldsymbol{r})
$$

We have obtained the following expression for the potential:

$$
\begin{gathered}
\varphi_{a b}(r)=\frac{e_{a} e_{b}}{r}\left(A \exp \left(-r k_{a b}\right)+B_{1} \exp \left(-r K_{1}\right)-B_{2} \exp \left(-r K_{2}\right)\right), \\
A=\frac{1-\beta}{\Delta}, B_{1,2}=\frac{C_{1,2}\left(C_{1,2}-\beta\right)}{\left(2 C_{2}-1\right) \Delta}, K_{1,2}=k_{a b} \sqrt{\frac{C_{2,1}}{\beta}} \\
\Delta=\gamma+\beta-1, C_{1,2}=\frac{1 \mp R a d}{2}, \operatorname{Rad}=\sqrt{1-4 \alpha} \\
\alpha=\frac{k_{D}^{4}}{k_{q}^{4}}, \quad \beta=\frac{k_{a b}^{2} k_{D}^{2}}{k_{q}^{4}}, \gamma=\frac{k_{D}^{2}}{k_{a b}^{2}} .
\end{gathered}
$$

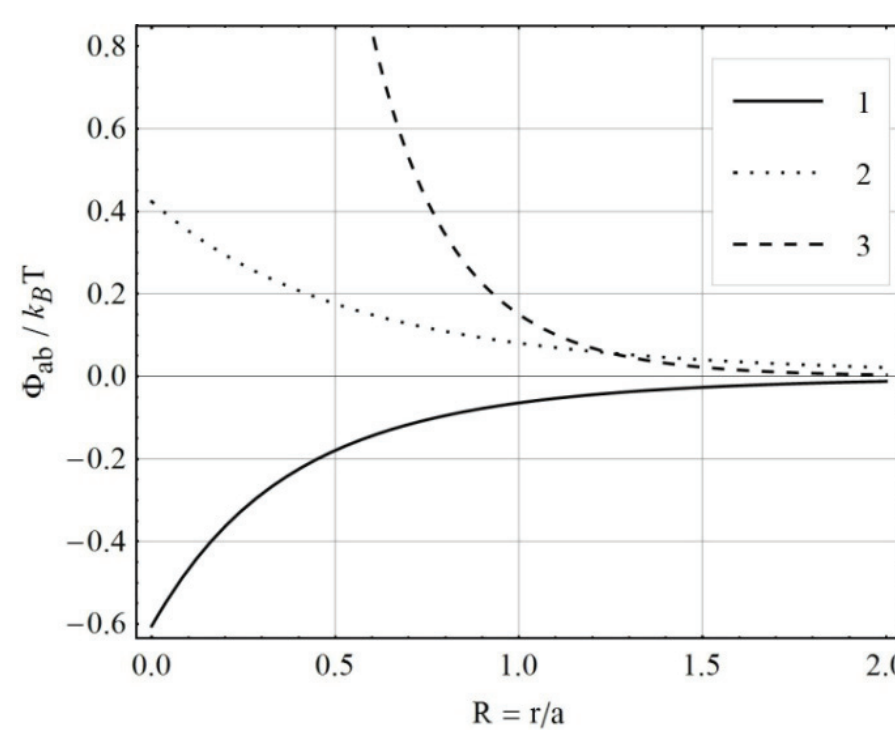

1 - electron-electron interaction; 2 - electron-proton interaction; 3 - proton-proton interaction at parameters

Figure 1 - The hydrogen plasma potentials (5) at $\Gamma=3, \mathrm{r}_{\mathrm{s}}=0.5$.

International Journal of Mathematics and Physics 5, №2, 48 (2014) 


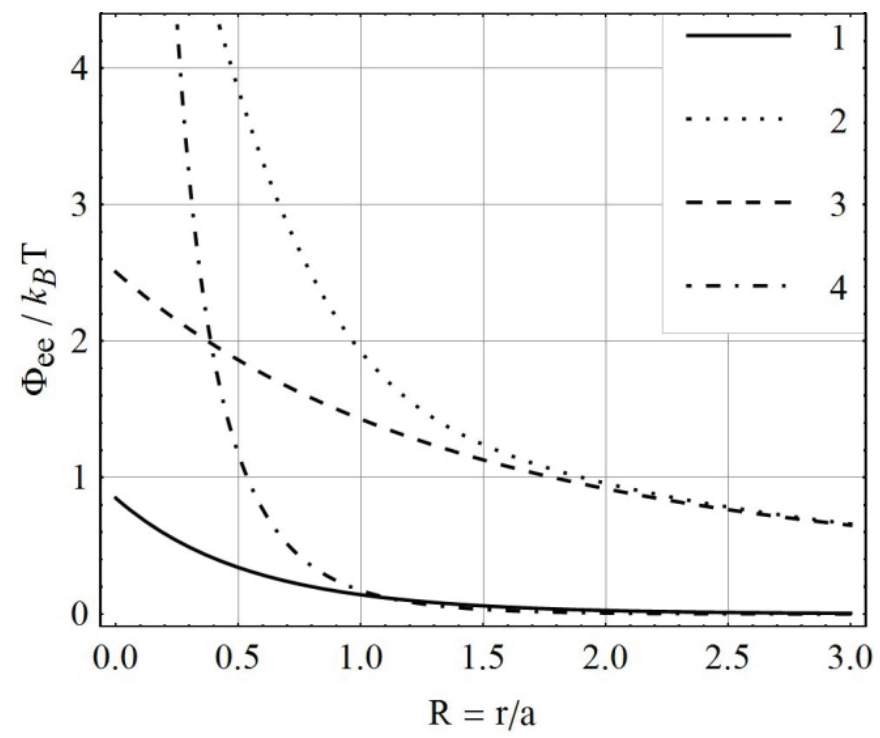

1 - potential (5); 2 - Kelbg potential; 3 - Deutsch potential; 4- Debye - Hueckel potential

Figure 2 - Potentials comparison.

\section{PlasmaPressure}

A system in the thermodynamic equilibrium can be described by using the measured macroscopic parameters, such as pressure and internal energy. The pressure of the plasma or equation of state (EOS) can be written in next way [1]:

$$
P=P_{i d}-\frac{2 \pi}{3} \int_{0}^{\infty} \sum_{a, b} n_{a} n_{b} \frac{d \varphi_{a b}(r)}{d r} g_{a b}(r) r^{3} d r,
$$

where $P_{i d}=\sum_{a} n_{a} k_{B} T$ is the pressure of the ideal gas.

We implemented this formula in order to calculate pressure of hot hydrogen plasma and compare with results of [7].

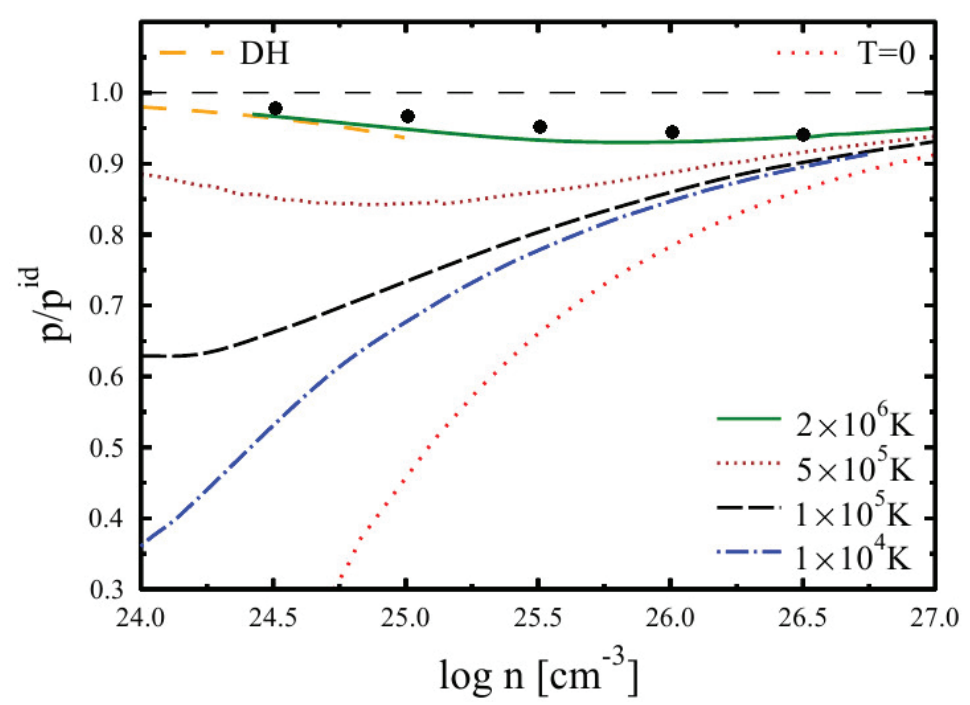

Figure3 - Pressure of hydrogenas predicted by the two-fluid model normalized by the ideal contribution [7], where black circles - formula (6) with potential (5) at $\mathrm{T}=2 * 10^{6} \mathrm{~K}$.

International Journal of Mathematics and Physics 5, №2, 48 (2014) 


\section{Conclusions}

An effective pair interaction potential of particles for ideal and weakly non-ideal plasma were obtained. It takes into account not only screening and dynamic quantum effects, but also statistical quantum effects. Comparison between other kinds of potentials was conducted. We used this potential for the calculation of plasma pressure and compared our result with [7]. It is in a good agreement at high temperatures.

\section{References}

1 Arkhipov Yu.V., Baimbetov F.B., Davletov A.E., Starikov K.V. Psevdopotentsialnaya teoriya plotnoy vysokotemperaturnoy plazmy. - Almaty: Qazaq Universiteti baspasy, 2002. - 111 p.

2 Adamyan V.M. and Tkachenko I.M. High frequency electrical conductivity of a collisional plasma // Teplofizika Vysokikh Temperatur. 1983. - Vol. 21. - P. 417-425.

3 Pierleoni C., Ceperley D.M., Bernu B., Magro W.R. Equation of state of the hydrogen plasma by pathintegral Monte Carlo simulation //Phys. Rev. Lett. - 1994. - Vol.73. - P.2145.

4 Hansen J.P., McDonald I.R. Microscopic simulation of a strongly coupled hydrogen plasma. // Phys. Rev. A. - 1981. - Vol. 23. - P.2041.

5 Riemann J., Schlanges M., DeWitt H.E., Kraeft W.D. Equation of state of the weakly degenerateone-component plasma // Physica A. 1995. - Vol. 219. - P.423.

6 Zerah G., Clerouin J., Pollock E.L. ThomasFermi molecular dynamics, linear screening and mean fieldtheories of plasmas //Phys. Rev. Lett. 1992. - Vol.69. - P.446.

7 Vorberger J. and Gericke D.O. The Hydrogen Equation of State at High Densities. // arXiv. $-1108.4826 \mathrm{v} 1$. 